\title{
Bloqueio Neuromuscular Residual após o Uso de Rocurônio ou Cisatracúrio *
}

\section{Residual Neuromuscular Block after Rocuronium or Cisatracurium}

Bruno Salomé de Morais ${ }^{1}$; Carlos Henrique Viana de Castro, TSA ${ }^{2}$; Vera Coelho Teixeira, TSA ${ }^{3}$; Alexandre Silva Pinto ${ }^{4}$

RESUMO
Morais BS, Castro CHV, Teixeira VC, Pinto AS - Bloqueio Neu-
romuscular Residual após o Uso de Rocurônio ou Cisatracúrio

JUSTIFICATIVA E OBJETIVOS: O bloqueio neuromuscular residual na sala de recuperação pós-anestésica (SRPA) é um fenômeno que pode aumentar a morbidade pós-operatória, com incidência variando entre $0 \%$ e $93 \%$. O objetivo deste estudo foi avaliar a incidência do bloqueio neuromuscular residual na SRPA.

MÉTODO: Foram estudados 93 pacientes submetidos à cirurgia geral com o uso de cisatracúrio ou rocurônio. Após a admissão na SRPA foi realizada a monitorização objetiva da função neuromuscular (aceleromiografia - TOF GUARD). O bloqueio neuromuscular residual foi definido como $S Q E<0,9$.

RESULTADOS: Do total de 93 pacientes, 53 receberam cisatracúrio e 40 rocurônio. As características demográficas, duração do procedimento e o uso de antagonista foram comparáveis em ambos os grupos. O bloqueio neuromuscular residual foi de $32 \%$ no subgrupo C (cisatracúrio) e 30\% no subgrupo $R$ (rocurônio). O bloqueio neuromuscular residual não foi associado à dose, idade, ou uso de antagonista, porém apresentou relação com a duração do procedimento cirúrgico. No subgrupo $C$ o tempo médio de cirurgia foi 135 minutos nos pacientes com bloqueio neuromuscular e 161 minutos nos pacientes sem bloqueio, $p<0,029$. No subgrupo $R$ o tempo médio de cirurgia foi 122 e 150 minutos, respectivamente, $p<$ 0,039 .

CONCLUSÕES: Os pacientes de ambos os grupos apresentaram incidência elevada de bloqueio neuromuscular residual na SRPA. A curarização residual pós-operatória continua um problema mesmo com os novos bloqueadores neuromusculares de ação intermediária. É de suma importância a monitorização objetiva de todos os pacientes submetidos à anestesia geral com uso de bloqueador neuromuscular.

\footnotetext{
* Recebido do (Received from) CET/SBA de Anestesiologia do Hospital Felício Rocho, Belo Horizonte, MG

1. Anestesiologista do Hospital Lifecenter e Hospital das Clínicas da UFMG

2. Diretor Clínico e Anestesiologista do Hospital Lifecenter. Especialista em Terapia Intensiva e Clínica Médica.

3. Anestesiologista e Responsável pelo CET/SBA do Hospital Felício Rocho. Especialista em Terapia Intensiva e Clínica Médica

4. Anestesiologista do Hospital Márcio Cunha.
}

Apresentado (Submitted) em 09 de fevereiro de 2005 Aceito (Accepted) para publicação em 11 de agosto de 2005

Endereço para correspondência (Correspondence to) Dr. Bruno Salomé de Morais

Rua Ajax Correa Rabelo, 160 Mangabeiras

30210-040 Belo Horizonte, MG

E-mail: brunomoraisanest@yahoo.com.br

(C) Sociedade Brasileira de Anestesiologia, 2005
Unitermos: BLOQUEADORES NEUROMUSCULARES:

cisatracúrio, rocurônio; MONITORIZAÇÃO: aceleromiografia, função neuromuscular; RECUPERAÇÃO PÓS- ANESTÉSICA: bloqueio neuromuscular residual

\section{SUMMARY}

Morais BS, Castro CHV, Teixeira VC, Pinto AS - Residual Neuromuscular Block after Rocuronium or Cisatracurium

BACKGROUND AND OBJECTIVES: Residual neuromuscular block in the post-anesthetic recovery unit (PACU) may increase postoperative morbidity from $0 \%$ to $93 \%$. This study aimed at evaluating the incidence of residual neuromuscular block in the PACU.

METHODS: Participated in this study 93 patients submitted to general anesthesia with cisatracurium or rocuronium. After PACU admission, neuromuscular function was objectively monitored (acceleromyography - TOF GUARD). Residual neuromuscular block was defined as TOF $<0.9$.

RESULTS: From 93 patients, 53 received cisatracurium and 40 rocuronium. Demographics, procedure length and the use of antagonists were comparable between groups. Residual neuromuscular block was $32 \%$ in subgroup C (cisatracurium) and $30 \%$ in subgroup $R$ (rocuronium). Residual neuromuscular block was unrelated to dose, age and use of antagonists, but was related to procedure length. In subgroup $C$, mean procedure length was 135 minutes for patients with neuromuscular block and 161 minutes for patients without $(p<0.029)$. In subgroup $R$, mean surgery length was 122 and 150 minutes, respectively $(p<0.039)$.

CONCLUSIONS: Both groups had high incidence of residual neuromuscular block in the PACU. Residual postoperative curarization is still a problem even with new intermediary action neuromuscular blockers. It is highly important to objectively monitor all patients submitted to general anesthesia with neuromuscular blockers.

Key Words: MONITORING: acceleromyography, neuromuscular function; NEUROMUSCULAR BLOCKERS: cisatracurium, rocuronium; POST-ANESTHETIC RECOVERY: residual neuromuscular block

\section{INTRODUÇÃO}

A paralisia ou fraqueza muscular pós-operatória decorArente da ausência ou incompleto antagonismo dos bloqueadores neuromusculares competitivos é conhecida como bloqueio neuromuscular residual pós-operatório (BNRPO). O BNRPO está associado a grande morbidade e mortalidade ${ }^{1,2}$ e sua incidência após a anestesia tem sido avaliada por diferentes autores há varias décadas, com ampla variação de resultados $(0 \% \text { a } 93 \%)^{3-6}$. Um estudo multicêntrico apontou que metade dos óbitos associados à anestesia eram devido à depressão respiratória pós-anestésica ${ }^{7}$. 
Este estudo teve por objetivo avaliar a incidência de bloqueio neuromuscular residual na sala de recuperação pós-anestésica entre os pacientes que receberam rocurônio ou cisatracúrio em dose única durante a realização de anestesia geral.

\section{MÉTODO}

Após aprovação do Comitê de Ética em Pesquisa da instituição e consentimento formal, todos os pacientes de ambos os sexos, estado físico ASA I ou II, com idade superior a 18 anos, admitidos na SRPA após anestesia geral com a utilização de rocurônio ou cisatracúrio para cirurgia geral, torácica, plástica, urológica, ginecológica, otorrinolaringológica, ortopédica ou neurológica, no período compreendido entre fevereiro e junho de 2003 foram incluídos no estudo. Foram excluídos os pacientes que tiveram a função neuromuscular monitorizada no intra-operatório ou que apresentavam qualquer distúrbio da função neuromuscular. Os pacientes admitidos na SRPA intubados, hipotérmicos (termostato posicionado na eminência tenar acusando temperatura inferior a $32^{\circ} \mathrm{C}$ ) ou que receberam qualquer outro bloqueador neuromuscular também não foram considerados para o estudo. Foram ainda excluídos todos os pacientes que receberam dose adicional de BNM durante a cirurgia, os que apresentavam alteração nos níveis de potássio, cálcio, magnésio ou que se recusaram a fazer parte do estudo.

Os pacientes foram divididos em dois subgrupos de acordo com o tipo de bloqueador neuromuscular utilizado: rocurônio (subgrupo R) e cisatracúrio (subgrupo C). Todos os pacientes foram monitorizados no momento de sua chegada na SRPA com cardioscópio, oxímetro digital e estimulador de nervo periférico além de receber oxigênio complementar por máscara facial num fluxo de $3 \mathrm{~L} \cdot \mathrm{min}^{-1}$.

A monitorização da função neuromuscular foi realizada por investigador que não participou do ato anestésico e não sabia quais drogas foram utilizadas. Este investigador era um médico em especialização (ME) em Anestesiologia escalado na SRPA. Todos os ME que participaram da coleta dos dados sabiam manipular o estimulador de nervo periférico e conheciam o protocolo de pesquisa. Estes investigadores não participaram do ato anestésico, portanto não fizeram escolha do bloqueador neuromuscular nem da condução da anestesia.

Nenhuma recomendação foi feita aos anestesiologistas, de modo que não houvesse influência quanto à técnica anestésica, monitorização neuromuscular ou reversão do bloqueio no intra-operatório.

Para a monitorização da função neuromuscular, foi utilizada a seqüência de quatro estímulos (SQE) com intensidade de $40 \mathrm{~mA}$ (aceleromiografia - TOF-GUARD Organon Teknika, NY) Os pacientes foram avaliados no momento da chegada na SRPA. A SQE foi aplicada três vezes com um intervalo de 30 segundos entre os estímulos e calculada a média entre estes três valores.

Foram considerados como pacientes com bloqueio neuromuscular residual aqueles que apresentaram $T_{4} / T_{1}<0,9$. Após a medida da SQE na SRPA, todos os pacientes com
$\mathrm{T}_{4} / \mathrm{T}_{1}$ entre 0,7 e 0,3 receberam $20 \mu \mathrm{g} \cdot \mathrm{kg}^{-1}$ de neostigmina e $10 \mu \mathrm{g} \cdot \mathrm{kg}^{-1}$ de atropina. Já aqueles pacientes com relação $\mathrm{T}_{4} / \mathrm{T}_{1}<0,3$ receberam $40 \mu \mathrm{g} \cdot \mathrm{kg}^{-1}$ de neostigmina e $20 \mu \mathrm{g} \cdot \mathrm{kg}^{-1}$ de atropina. Os pacientes que apresentaram menos de duas respostas $\left(\mathrm{T}_{2}\right)$ a SQE foram re-intubados.

Também foi analisado o peso do paciente, idade, período entre a utilização do BNM e a medida da SQE, dose do bloqueador neuromuscular utilizado, uso de fármacos que interferem no bloqueio neuromuscular e utilização de neostigmina durante a cirurgia. As comparações entre os grupos em relação às variáveis contínuas de interesse foram realizadas utilizando-se o teste de Mann-Whitney. Para comparação em relação às variáveis categóricas utilizou-se o teste Qui-quadrado. Nos casos em que ocorreram valores esperados menores que 5 utilizou-se o teste Exato de Fisher. O nível de significância estatística adotado foi de $5 \%$.

\section{RESULTADOS}

Foram avaliados 93 pacientes no período compreendido entre $27 / 02 / 03$ e 02/06/03, sendo 45 do sexo masculino e 48 do sexo feminino. Deste total de pacientes, 47 eram estado físico ASA I e 46 ASA II. Cinqüenta e três pacientes receberam cisatracúrio e 40 rocurônio. Todos os pacientes foram submetidos à anestesia geral balanceada para realização de procedimento cirúrgico conforme a tabela I.

Tabela I - Distribuição das Cirurgias por Diferentes Clínicas

\begin{tabular}{lcc}
\hline Clínicas & Freqüência & Percentual \\
\hline Otorrinolaringologia & 26 & 27,9 \\
Cirurgia geral & 39 & 41,9 \\
Ginecologia & 7 & 7,6 \\
Cirurgia plástica & 6 & 6,5 \\
Ortopedia & 5 & 5,4 \\
Urologia & 4 & 4,3 \\
Neurocirurgia & 3 & 3,2 \\
Cirurgia torácica & 3 & 3,2 \\
\hline Total & 93 & 100 \\
\hline
\end{tabular}

A idade média dos pacientes foi 47,8 anos e o peso médio $66,7 \mathrm{~kg}$. Apenas 28 pacientes (30\%) receberam neostigmina ao término do procedimento.

A tabela II mostra a comparação entre os dois subgrupos quanto à idade, peso, sexo, estado físico (ASA), número de DE95 utilizado, duração do procedimento, uso de neostigmina e de algum medicamento que pudesse potencializar o BNM.

No subgrupo C, 17 pacientes (32\%) apresentaram bloqueio neuromuscular residual enquanto no subgrupo $R$ a incidência foi de 12 pacientes $(30 \%),(p=0,83)$. No subgrupo $\mathrm{C}$ a dose média administrada do bloqueador neuromuscular foi $0,1575 \mathrm{mg} \cdot \mathrm{kg}^{-1}$. (DP 0,048). Esta dose eqüivale a 3,15 DE95 para o cisatracúrio. No subgrupo $R$ a dose média utilizada foi $0,66 \mathrm{mg} \cdot \mathrm{kg}^{-1}$, (DP 0,157). Esta dose eqüivale a 2,20 DE95 para o rocurônio. A proporção de pacientes que recebeu dose de bloqueador neuromuscular superior a 3 DE95 foi de 
$47 \%$ no subgrupo C e $5 \%$ no subgrupo $R(p<0,001)$. O percentual de pacientes que fez uso de neostigmina ao término da anestesia foi de $30,2 \%$ no subgrupo $\mathrm{C}$ e $30 \%$ no subgrupo $\mathrm{R}$. Dentre os pacientes que receberam neostigmina, a dose média administrada no subgrupo $C$ foi $0,027 \mathrm{mg}^{\mathrm{kg}}{ }^{-1}$ (DP 0,0078 ) e $0,029 \mathrm{mg} \cdot \mathrm{kg}^{-1}$ no subgrupo $\mathrm{R}(\mathrm{DP} 0,0068) \operatorname{com~} \mathrm{p}=$ 0,625 . A dose do BNM utilizado em dose única não apresentou relação com o grau de curarização residual, conforme a tabela III.

Tabela II - Características Demográficas dos Pacientes nos Diferentes Subgrupos

\begin{tabular}{lccc}
\hline Variáveis & Cisatracúrio & Rocurônio & $\mathrm{p}$ \\
\hline Idade média (anos) & 46 & 50,18 & 0,753 \\
Peso médio $(\mathrm{kg})$ & 70,23 & 64,25 & 0,149 \\
Sexo feminino & $45,1 \%$ & $56,4 \%$ & 0,126 \\
Estado físico & & & \\
$\quad$ ASA I & $49 \%$ & $52,50 \%$ & 0,835 \\
$\quad$ ASA II & $51 \%$ & $47,50 \%$ & 0,835 \\
Número de DE95 & 3,16 & 2,2 & 0,001 \\
Duração média da cirurgia (min) & 152,92 & 144,87 & 0,889 \\
Uso de neostigmina & $30,20 \%$ & $30 \%$ & 0,984 \\
Drogas que potencializam o BNM & $24 \%$ & $20 \%$ & 0,269 \\
\hline
\end{tabular}

Tabela III - Relação entre a Dose de Rocurônio e Cisatracúrio com Valores da SQE

\begin{tabular}{lcccccc}
\hline Grupos & SQE & $\mathrm{n}$ & $\begin{array}{c}\text { Média } \\
(\mathrm{mg})\end{array}$ & Mediana & Desvio-Padrão & $\mathrm{p}$ \\
\hline Rocurônio & SQE $<90$ & 12 & 44,17 & 50,00 & 9,00 & 0,391 \\
& $\mathrm{SQE} \geq 90$ & 28 & 41,70 & 45,00 & 10,48 & \\
Cisatracúrio & $\mathrm{SQE}<90$ & 17 & 12,71 & 12,00 & 4,37 & 0,086 \\
& $\mathrm{SQE} \geq 90$ & 36 & 10,38 & 10,00 & 2,56 & \\
\hline
\end{tabular}

A tabela IV mostra o intervalo de tempo entre a administração do BNM e medida da SQE nos diferentes grupos. A tabela $V$ representa a relação entre SQE e uso de neostigmina nos diferentes subgrupos.
Tabela V - Relação entre a SQE e o Uso de Neostigmina

\begin{tabular}{lccccc}
\hline Grupos & SQE & Antagonista & Freqüência & Porcentagem & $\mathrm{p}$ \\
\hline Cisatracúrio & SQE & Não & 13 & 76,5 & 0,449 \\
& $<90$ & Sim & 4 & 23,5 & \\
& SQE & Não & 24 & 66,7 & \\
& $\geq 90$ & Sim & 12 & 33,3 & \\
Rocurônio & SQE & Não & 10 & 83,3 & 0,176 \\
& $<90$ & Sim & 2 & 16,7 & \\
& SQE & Não & 18 & 64,3 & \\
& $\geq 90$ & Sim & 10 & 35,7 & \\
\hline
\end{tabular}

Dos pacientes que receberam neostigmina ao término do procedimento, não houve diferença na dose média utilizada em relação aqueles que apresentaram ou não bloqueio neuromuscular residual. No subgrupo $\mathrm{C}$, os pacientes com BNRPO receberam 0,21 mg. $\mathrm{kg}^{-1}$ (DP 0,0049) de neostigmina e aqueles sem bloqueio neuromuscular residual receberam $0,29 \mathrm{mg} \cdot \mathrm{kg}^{-1}$ (DP 0,0077) com $\mathrm{p}=0,059$. Já no subgrupo $\mathrm{R}$, os pacientes com BNRPO receberam $0,025 \mathrm{mg} \cdot \mathrm{kg}^{-1}$ (DP 0,0002 ) de neostigmina e aqueles sem BNRPO 0,03 mg. $\mathrm{kg}^{-1}$ (DP 0,0072 ) com $p=0,449$. Porém quando os pacientes foram subdivididos em relação à dose de neostigmina utilizada, notou-se menor incidência de bloqueio neuromuscular residual entre aqueles que receberam dose superior a 0,03 $\mathrm{mg} \cdot \mathrm{kg}^{-1}$, conforme a tabela VI.

Tabela VI - SQE de acordo com a Dose de Neostigmina Utilizada

\begin{tabular}{lccc}
\hline Dose de Neostigmina & \multicolumn{2}{c}{ SQE } & Total \\
\hline & $<90$ & $\geq 90$ & \\
\hline$\leq 0,03$ & 6 & 9 & 15 \\
$>0,03$ & 0 & 13 & 13 \\
Total & 6 & 22 & 28 \\
\hline
\end{tabular}

$p=0,0178$

A tabela VII mostra a relação entre os valores da SQE e no grupo de pacientes que utilizou alguma droga que teoricamente potencializa do efeito do bloqueador neuromuscular não-despolarizante.

Tabela IV - Correlação da SQE com o Tempo de Administração do BNM

\begin{tabular}{|c|c|c|c|c|c|c|c|c|}
\hline & \multicolumn{5}{|c|}{ Cisatracúrio } & \multicolumn{3}{|c|}{ Rocurônio } \\
\hline & $\mathrm{n}$ & Média & Mediana & $\mathrm{DP}$ & $\mathrm{n}$ & Média & Mediana & $\mathrm{DP}$ \\
\hline $\mathrm{SQE}<90$ & 17 & 135,00 & 120,00 & 63,40 & 12 & 122,08 & 125,00 & 33,88 \\
\hline $\mathrm{SQE} \geq 90$ & 36 & 161,39 & 150,00 & 51,75 & 28 & 154,64 & 150,00 & 44,51 \\
\hline$p$ & & & 0,028 & & & & 0,038 & \\
\hline
\end{tabular}


Tabela VII - Relação entre a SQE e o Uso de Medicamentos que Potencializam os BNM

\begin{tabular}{lcccc}
\hline Grupos & $\mathrm{SQE}$ & Freqüência & Porcentagem & $\mathrm{p}$ \\
\hline Cisatracúrio & $\mathrm{SQE}<90$ & 3 & 17,6 & 0,39 \\
& $\mathrm{SQE} \geq 90$ & 10 & 27,8 & \\
Rocurônio & $\mathrm{SQE}<90$ & 2 & 16,7 & 0,88 \\
& $\mathrm{SQE} \geq 90$ & 4 & 14,8 & \\
\hline
\end{tabular}

Três pacientes do subgrupo $\mathrm{C}$ e dois do subgrupo $\mathrm{R}$ apresentaram SQE entre 0,3 e 0,7 pelo que foram medicados com $0,02 \mathrm{mg} \cdot \mathrm{kg}^{-1}$ de neostigmina e $0,02 \mathrm{mg} \cdot \mathrm{kg}^{-1}$ de atropina. Um paciente do subgrupo $\mathrm{C}$ apresentou $\mathrm{SQE}=0,25$, pelo que recebeu $0,04 \mathrm{mg} \cdot \mathrm{kg}^{-1}$ de neostigmina e $0,02 \mathrm{mg} \cdot \mathrm{kg}^{-1}$ de atropina. Após 10 minutos foi realizada nova medida e todos estes pacientes apresentaram SQE superior a 0,9. Nenhum paciente necessitou ser re-intubado.

\section{DISCUSSÃO}

Em 1942 Griffth e Johnson descreveram em publicação pioneira o uso do curare em anestesia geral ${ }^{8}$. Hoje passados mais de 60 anos desde a introdução dos curares em anestesia, os bloqueadores neuromusculares constituem um dos pontos vitais da anestesia moderna pois facilitam a intubação traqueal, proporcionam relaxamento do campo operatório, permitem imobilização e facilitam o controle da ventilação. Porém um dos principais efeitos colaterais deste grupo de drogas é a possibilidade do estabelecimento de bloqueio neuromuscular residual.

É fundamental a observação próxima e contínua após a reversão. A possibilidade de bloqueio neuromuscular residual deve sempre ser lembrada na sala de recuperação pós-anestésica (SRPA), já que raramente é possível demonstrar uma recuperação neuromuscular plena baseada apenas em parâmetros clínicos. Os sinais clínicos de ausência de bloqueio neuromuscular residual (levantar e manter a cabeça por cinco segundos, expor a língua, abrir os olhos, tossir e inspirar profundamente) mostraram resultados falso-negativos em todos os grupos quando comparados à estimulação nervosa periférica ${ }^{3}$. Nas situações duvidosas é fundamental que seja realizado o registro através de métodos objetivos.

De acordo com o que se deseja analisar, várias formas de estimulação podem ser aplicadas: estímulo isolado (EI), seqüência de quatro estímulos (SQE) também conhecido como TOF (train of four), estimulação tetânica (ET), estimulação com contagem pós-tetânica (CPT), estimulação em dupla salva (EDS) e salvas de tétano pós-tétano (TPT). Para monitorização pós-operatória da função neuromuscular podem ser utilizados: a SQE e oEDS. Aseqüência de quatro estímulos é a mais comumente utilizada por apresentar as seguintes vantagens: não dói, não exige medida de controle além de refletir adequadamente o grau de bloqueio neuromuscular.
O bloqueio neuromuscular residual é um fator de risco para complicações pulmonares pós-operatórias como aspiração, pneumonia e atelectasia $^{2,9}$.

Apesar da tendência de maior uso dos bloqueadores neuromusculares (BNM) de ação intermediária, a incidência de bloqueio neuromuscular residual continua elevada mesmo com utilização de anticolinesterásicos ${ }^{4,5}$.

Estudo recente apontou que $24,7 \%$ dos pacientes que receberam vecurônio e $14,7 \%$ dos que receberam rocurônio apresentaram SQE $<0,7$ na SRPA, após a tentativa de reversão do bloqueio neuromuscular com piridostigmina ${ }^{5}$.

Os estimuladores mais simples permitem apenas a avaliação visual ou tátil da contração muscular. Contudo, diversos estudos sugerem que mesmo em mãos experientes pode ser difícil detectar fadiga por esses métodos quando $T_{4} / T_{1}>0,3$ 10,11. A utilização do estímulo em dupla salva facilita a detecção visual ou tátil de fadiga, mas também apresenta limitações ${ }^{11}$. O valor $T_{4} / T_{1}>0,7$ sugere que o paciente não desenvolverá dificuldade respiratória significativa; entretanto, não é suficiente para prevenir aspiração do conteúdo gástrico ou obstrução de vias aéreas ${ }^{12}$. Adificuldade de deglutição desaparece apenas com a relação $T_{4} / T_{1}>0,9^{13}$. Na presença da $\mathrm{SQE}<0,9$ no adutor do polegar, a proteção das vias aéreas está prejudicada, aumentando em quatro a cinco vezes o risco de aspiração ${ }^{9}$. Para assegurar a função muscular normal e a regulação ventilatória, a SQE $>0,9$ no adutor do polegar deve ser alcançado antes do paciente ser extubado ${ }^{9}$.

Diversos estudos que padronizaram bloqueio neuromuscular residual como quando $\mathrm{SQE}<0,7$ demonstraram incidência de $8 \%$ a $42 \%$ com o vecurônio ${ }^{1}, 24 \%$ com o mivacúrio ${ }^{14}$, e até $65 \%$ com o atracúrio. Considerando-se que o valor seguro da SQE para extubação seja superior a 0,9 a incidência de bloqueio neuromuscular residual é ainda maior do que a encontrada naqueles estudos.

O subgrupo $C$ recebeu maior dose relativa de BNM $(3,15$ DE95) que o subgrupo R (2,2 DE95). Esta diferença talvez tenha ocorrido pela maior latência do cisatracúrio quando comparada a do rocurônio, com uso de maior dose relativa para obtenção de boas condições de intubação em curto período de tempo.

Em ambos os subgrupos ocorreram elevada incidência de bloqueio neuromuscular residual pós-operatório, não havendo diferença estatística significativa no bloqueio neuromuscular residual entre o cisatracúrio e o rocurônio. A incidência de bloqueio neuromuscular na SRPA foi de $32 \%$ no subgrupo $\mathrm{C}$ e $30 \%$ no subgrupo R. A incidência de bloqueio neuromuscular residual manteve-se elevada, em níveis equivalentes aos resultados obtidos em estudos conduzidos nas últimas décadas.

Não houve diferença estatística significativa em relação à dose do BNM entre os pacientes que apresentaram BNRPO, mostrando que o bloqueador neuromuscular utilizado em dose única não foi determinante do bloqueio neuromuscular residual. O aumento da dose do cisatracúrio prolonga o período de bloqueio neuromuscular profundo (sem resposta a neuroestimulação). Entretanto, ao iniciar a recuperação espontânea, o perfil apresentado é semelhante, apesar da dife- 
rença de dose, permitindo, desta maneira, predizer o tempo de recuperação ${ }^{15}$. Estudo semelhante concluiu que a dose do atracúrio não está associada a BNRPO ${ }^{4}$. Outro autor verificou que maiores doses de vecurônio estavam associadas a maior risco de BNRPO ${ }^{1}$, porém neste estudo foram utilizadas doses múltiplas do BNM.

Existe diferença estatística significativa entre o tempo de administração do BNM e a SQE em ambos subgrupos (Tabela IV). O menor intervalo entre a administração do BNM e término do procedimento está relacionado à maior incidência de bloqueio neuromuscular residual, por isto os pacientes submetidos a procedimentos de curta duração estão sob maior risco. Resultados semelhantes foram encontrados com o vecurônio ${ }^{1}$, atracúrio ${ }^{4}$ e rocurônio ${ }^{5}$.

Não houve diferença estatística quanto ao bloqueio neuromuscular entre os pacientes que receberam neostigmina ou não (Tabela $V$ ). A neostigmina é capaz de antagonizar o bloqueio neuromuscular eficazmente ${ }^{16}$. As controvérsias existem no que diz respeito às doses necessárias de neostigmina para reversão completa, pois elas dependem do fármaco utilizado, da sua dose, do tempo decorrido desde a sua administração e do uso concomitante de drogas que podem interferir com os bloqueadores neuromusculares.

Em todos os pacientes o período entre a administração da neostigmina e a avaliação do bloqueio neuromuscular residual foi superior a 10 minutos.

Nos subgrupos cisatracúrio e rocurônio a dose média empregada de neostigmina foi relativamente pequena, 0,027 $\mathrm{mg} \cdot \mathrm{kg}^{-1}$ e $0,029 \mathrm{mg} \cdot \mathrm{kg}^{-1}$, respectivamente. As doses médias de neostigmina utilizadas não foram suficientes para reverter o bloqueio neuromuscular provocado pelo cisatracúrio e rocurônio, uma vez que não foi encontrada diferença estatística significativa no valor da SQE entre aqueles pacientes que receberam antagonista ou não. Estudos apontam que a dose de $0,05 \mathrm{mg} \cdot \mathrm{kg}^{-1}$ de neostigmina é eficaz na reversão do bloqueio neuromuscular promovido pelo vecurônio ${ }^{1,16}$.

Quando os pacientes foram subdivididos em relação à dose de neostigmina utilizada, evidenciou-se que a dose superior a $0,03 \mathrm{mg} \cdot \mathrm{kg}^{-1}$ foi eficaz na reversão do bloqueio neuromuscular, sendo que todos os pacientes que receberam esta dose chegaram na SRPAcom SQE superior a 0,9 (Tabela VI) Diversas drogas podem interagir com os bloqueadores neuromusculares, alterando o padrão do bloqueio. O estudo não foi sensível em mostrar esta interação medicamentosa visto que não houve diferença estatística significativa nos valores da SQE entre aqueles pacientes que usaram estas drogas ou não.

A utilização de subdose de neostigmina e procedimentos de menor duração está associada a maior risco de BNRPO. É fundamental a observação cuidadosa e contínua na SRPA, mesmo após a reversão.

A monitorização objetiva da função neuromuscular deve ser sempre utilizada, pois possibilita a decisão correta sobre a necessidade de reversão, dose de reversão e a eficiência do antagonismo.

Os pacientes de ambos os grupos apresentaram incidência elevada de bloqueio neuromuscular residual na SRPA. O blo- queio neuromuscular residual pós-operatório continua um problema, mesmo com os novos bloqueadores neuromusculares de ação intermediária

\section{Residual Neuromuscular Block after Rocuronium or Cisatracurium}

Bruno Salomé de Morais, M.D.; Carlos Henrique Viana de Castro, TSA, M.D.; Vera Coelho Teixeira, TSA, M.D.; Alexandre Silva Pinto, M.D.

\section{INTRODUCTION}

Postoperative paralysis or muscle weakness due to lack of, or incomplete antagonism of don-depolarizing muscle relaxants is known as residual postoperative curarization (RPOC). RPOC is associated to major morbidity and mortality ${ }^{1,2}$. The incidence of post-anesthetic residual neuromuscular block has been evaluated by different authors for decades, with broad difference in results $(0 \% \text { to } 93 \%)^{3-6}$. A multicenter study has observed that half the deaths associated to anesthesia were due to post-anesthetic respiratory depression ${ }^{7}$.

This study aimed at evaluating the incidence of residual neuromuscular block in the post-anesthetic recovery unit in patients receiving rocuronium or cisatracurium during general anesthesia.

\section{METHODS}

After the Research Ethics Committee approval and their informed consent, participated in this study all patients of both genders, physical status ASA I or II, aged above 18 years, admitted to the PACU after general anesthesia with rocuronium or cisatracurium for general, thoracic, plastic, urologic, gynecologic, ENT, orthopedic or neurologic surgery in the period Feb-Jun/2003. Exclusion criteria were patients with neuromuscular function intraoperatively monitored or those with any neuromuscular function disorder. Patients admitted to the PACU intubated, hypothermal (probe positioned at the tenar eminence recording temperatures below $32^{\circ} \mathrm{C}$ ) or receiving any other neuromuscular blocker were also excluded from the study. Additional exclusion criteria were patients receiving additional NMB dose during surgery, those with altered potassium, calcium or magnesium levels, and those refusing to be part of the study.

Patients were divided in two subgroups according to the neuromuscular blocker: rocuronium (subgroup $\mathrm{R}$ ) and cisatracurium (subgroup $\mathrm{C}$ ). All patients were monitored at PACU arrival with cardioscopy, pulse oximetry and peripheral nerve stimulator, in addition to receiving oxygen under facial mask in 3 L. $\min ^{-1}$ flow.

Neuromuscular function monitoring was performed by investigator not participating in the anesthetic induction and blind Vol. 55, Nº 6, Novembro - Dezembro, 2005 
to the drugs used. This investigator was a physician specializing in Anesthesiology allocated to the PACU. All physicians participating in data collection knew how to handle peripheral nerve stimulators and were familiar with the research protocol. These investigators have not participated in the anesthetic induction, so they have not chosen the neuromuscular blocker or the anesthetic approach.

No recommendation was made to anesthesiologists in order not to influence anesthetic technique, neuromuscular monitoring or intraoperative blockade reversal.

TOF stimulation with intensity of $40 \mathrm{~mA}$ was used to monitor neuromuscular function (acceleromyography-TOF-GUARD Organon Teknika, NY). Patients were evaluated at PACU arrival. TOF was applied three times with 30 -second intervals between stimulations and the mean of these three values was calculated.

Patients with residual neuromuscular block were those with $\mathrm{T}_{4} / \mathrm{T}_{1}<0.9$. After TOF measurement in the PACU, all patients with $\mathrm{T}_{4} / \mathrm{T}_{1}$ between 0.7 and 0.3 received $20 \mu \mathrm{g} . \mathrm{kg}^{-1}$ neostigmine and $10 \mu \mathrm{g} . \mathrm{kg}^{-1}$ atropine. Patients with $\mathrm{T}_{4} / \mathrm{T}_{1}<0.3$ received $40 \mu \mathrm{g} \cdot \mathrm{kg}^{-1}$ neostigmine and $20 \mu \mathrm{g} \cdot \mathrm{kg}^{-1}$ atropine. Patients with less than two TOF responses $\left(\mathrm{T}_{2}\right)$ were re-intubated.

Patients' weight, age, period between NMB and TOF, neuromuscular blocker dose, use of drugs interfering with neuromuscular block and administration of neostigmine during surgery were also evaluated. Mann-Whitney test was used for comparisons between groups with regard to continuous variables. Chi-square test was used for categorical variables. Fisher Exact test was used when expected values were below 5. Statistical significance level was $5 \%$.

\section{RESULTS}

The study consisted in the evaluation of 93 patients in the period $02 / 27 / 03$ to $06 / 02 / 03$, being 45 males and 48 females. From these, 47 patients were physical status ASA I and 46 were ASA II. Cisatracurium was administered to 53 patients and rocuronium to 40 . All patients were submitted to balanced general anesthesia to be submitted to surgical procedures as shown in table I.

Table I - Distribution of Procedures by Different Clinics

\begin{tabular}{lcc}
\hline Clinics & Frequency & Percentage \\
\hline ENT & 26 & 27.9 \\
General surgery & 39 & 41.9 \\
Gynecology & 7 & 7.6 \\
Plastic surgery & 6 & 6.5 \\
Orthopedics & 5 & 5.4 \\
Urology & 4 & 4.3 \\
Neurosurgery & 3 & 3.2 \\
Thoracic surgery & 3 & 3.2 \\
\hline Total & 93 & 100 \\
\hline
\end{tabular}

Mean patients' age was 47.8 years and mean weight was 66.7 $\mathrm{kg}$. Only 28 patients $(30 \%)$ received neostigmine at the end of surgery.

Table II shows the comparison between groups in age, weight, gender, physical status (ASA), number of DE95 used, procedure length, use of neostigmine and any drug potentially exacerbating NMB.

Table II - Patients' Demographics in Different Subgroups

\begin{tabular}{lccc}
\hline Variables & Cisatracurium & Rocuronium & $\mathrm{p}$ \\
\hline Mean age (years) & 46 & 50.18 & 0.753 \\
Mean weight $(\mathrm{kg})$ & 70.23 & 64.25 & 0.149 \\
Female & $45.1 \%$ & $56.4 \%$ & 0.126 \\
Physical status & & & \\
$\quad$ ASA I & $49 \%$ & $52.50 \%$ & 0.835 \\
$\quad$ ASA II & $51 \%$ & $47.50 \%$ & 0.835 \\
Number of DE95 & 3.16 & 2.2 & 0.001 \\
Mean surgery length (min) & 152.92 & 144.87 & 0.889 \\
Use of neostigmine & $30.20 \%$ & $30 \%$ & 0.984 \\
Drugs potentiating NMB & $24 \%$ & $20 \%$ & 0.269 \\
\hline
\end{tabular}

In subgroup C, 17 patients (32\%) presented residual neuromuscular block, while in subgroup $R$ the incidence was 12 patients $(30 \%)(p=0.83)$. Mean neuromuscular block dose for subgroup $C$ was $0.1575 \mathrm{mg} \cdot \mathrm{kg}^{-1}$ (SD 0.048). This dose is equivalent to $3.15 \mathrm{DE} 95$ for cisatracurium. In subgroup $\mathrm{R}$, mean dose was $0.66 \mathrm{mg} \cdot \mathrm{kg}^{-1}$ (SD 0.157). This dose is equivalent to 2.20 DE95 for rocuronium. Patients receiving neostigmine at the end of anesthesia were $30.2 \%$ in subgroup $C$ and $30 \%$ in subgroup R. Among patients receiving neostigmine, mean dose for subgroup $\mathrm{C}$ was $0.027 \mathrm{mg} . \mathrm{kg}^{-1}$ (SD 0.0078) and $0.029 \mathrm{mg} \cdot \mathrm{kg}^{-1}$ for subgroup R (SD 0.0068) with $p=0.625$. Bolus NMB dose was not related to residual curarization as shown in table III.

Table III - Relation of Rocuronium and Cisatracurium Dose and TOF Values

\begin{tabular}{lcccccc}
\hline Groups & TOF & $\mathrm{n}$ & $\begin{array}{c}\text { Mean } \\
(\mathrm{mg})\end{array}$ & Median & $\begin{array}{c}\text { Standard } \\
\text { Deviation }\end{array}$ & $\mathrm{p}$ \\
\hline Rocuronium & TOF $<90$ & 12 & 44.17 & 50.00 & 9.00 & 0.391 \\
& TOF $\geq 90$ & 28 & 41.70 & 45.00 & 10.48 & \\
Cisatracurium & TOF $<90$ & 17 & 12.71 & 12.00 & 4.37 & 0.086 \\
& TOF $\geq 90$ & 36 & 10.38 & 10.00 & 2.56 & \\
\hline
\end{tabular}

Table IV shows time elapsed between NMB administration and TOF measurement in both groups. Table $V$ represents the ratio between TOF and use of neostigmine in different subgroups. 
Table IV - Correlation of TOF and NMB Administration Time

\begin{tabular}{lcccccccc}
\hline & \multicolumn{3}{c}{ Cisatracurium } & \multicolumn{3}{c}{ Rocuroniun } \\
\hline TOF $<90$ & $\mathrm{n}$ & Mean & Median & SD & $\mathrm{n}$ & Mean & Median & SD \\
TOF $\geq 90$ & 17 & 135.00 & 120.00 & 63.40 & 12 & 122.08 & 125.00 & 33.88 \\
$\mathrm{p}$ & 36 & 161.39 & 150.00 & 51.75 & 28 & 154.64 & 150.00 & 44.51 \\
\hline
\end{tabular}

Table V - Relation between TOF and Neostigmine Administration

\begin{tabular}{lccccc}
\hline Groups & TOF & Antagonist & Frequency & Percentage & $p$ \\
\hline Cisatracurim & TOF & No & 13 & 76.5 & 0.449 \\
& $<90$ & Yes & 4 & 23.5 & \\
& TOF & No & 24 & 66.7 & \\
\cline { 2 - 3 } Rocuronium & $\geq 90$ & Yes & 12 & 33.3 & \\
& TOF & No & 10 & 83.3 & 0.176 \\
& $<90$ & Yes & 2 & 16.7 & \\
& TOF & No & 18 & 64.3 & \\
& $\geq 90$ & Yes & 10 & 35.7 & \\
\hline
\end{tabular}

Among patients receiving neostigmine at the end of surgery there has been no difference in mean dose with regard to patients with or without residual neuromuscular block. In subgroup C, patients with RPOC received $0.21 \mathrm{mg}^{\mathrm{kg}}{ }^{-1}$ (SD 0.0049 ) neostigmine and those without residual curarization received $0.029 \mathrm{mg} \cdot \mathrm{kg}^{-1}$ (SD 0.0077 ) neostigmine, with $\mathrm{p}=$ 0.059 . In subgroup $R$, patients with RPOC received 0.025 $\mathrm{mg} \cdot \mathrm{kg}^{-1}$ (SD 0.0002) neostigmine while those without RPOC received $0.03 \mathrm{mg} \cdot \mathrm{kg}^{-1}$ (SD 0.0072 ) with $p=0.449$. However, when patients were subdivided with regard to neostigmine dose, there has been a lower incidence of residual curarization among those receiving more than $0.03 \mathrm{mg} \cdot \mathrm{kg}^{-1}$, as shown in table VI.

Table VI - TOF according to Neostigmine Dose

\begin{tabular}{lccc}
\hline Neostigmine Dose & \multicolumn{2}{c}{ TOF } & Total \\
\hline & $<90$ & $\geq 90$ & \\
\hline 0.03 & 6 & 9 & 15 \\
$>0.03$ & 0 & 13 & 13 \\
Total & 6 & 22 & 28 \\
\hline
\end{tabular}

$p=0.0178$

Table VII shows the relation between TOF values and concomitant use of some drug exacerbating competitive neuromuscular blocker effect.

Three subgroup $C$ patients and two subgroup $R$ patients presented TOF between 0.3 and 0.7 and were medicated with $0.002 \mathrm{mg}$ neostigmine and $0.02 \mathrm{mg} . \mathrm{kg}^{-1}$ atropine. After $10 \mathrm{mi}-$ nutes a new measurement was performed and all patients presented TOF above 0.9. No patient needed re-intubation.
Table VII - Relation between TOF and Drugs Potentiating NMB

\begin{tabular}{lcccc}
\hline Groups & TOF & Frequency & Percentage & $\mathrm{p}$ \\
\hline Cisatracurium & TOF $<90$ & 3 & 17.6 & 0.39 \\
& TOF $\geq 90$ & 10 & 27.8 & \\
Rocuronium & TOF $<90$ & 2 & 16.7 & 0.88 \\
& TOF $\geq 90$ & 4 & 14.8 & \\
\hline
\end{tabular}

\section{DISCUSSION}

In 1942, Griffith and Johnson described, in a pioneer publication, the use of curare in general anesthesia ${ }^{8}$. Today, more than 60 years after the introduction of curare in anesthesiology, neuromuscular blockers are a landmark for modern anesthesia since they help tracheal intubation, provide operating field relaxation, allow for immobilization and help ventilation control. However, a major side effect of this group of drugs is the possibility of residual neuromuscular block.

Close and continuous observation after reversal is critical. Residual neuromuscular block should always be reminded in the post-anesthetic recovery unit (PACU), since it is seldom possible to show total neuromuscular recovery based on clinical parameters only. Clinical signs of lack of residual curarization (raise and maintain the head for 5 seconds, show the tongue, open eyes, cough and deeply breathe) have provided false-negative results in all groups when compared to peripheral nerve stimulation ${ }^{3}$. In dubious situations it is critical to use more objective methods.

According to what is to be analyzed, several stimulation methods may be applied: isolated stimulation (IS), train of four (TOF), stimulation with post-tetanus count (PTC), double burst stimulation (DBS) and tetanus post-tetanus bursts (TPT). The following may be used for postoperative neuromuscular function monitoring: TOF and DBS. Train of four is the most common for the following advantages: it is painless, does not require control measures and adequately reflects neuromuscular block level.

Residual neuromuscular block is a risk factor for postoperative pulmonary complications such as aspiration, pneumonia and atelectasis ${ }^{2,9}$.

Although the trend for more frequent use of neuromuscular blockers (NMB) of intermediary action, the incidence of residual neuromuscular block is still high, even with the administration of anti-cholinesterase drugs ${ }^{4,5}$.

Revista Brasileira de Anestesiologia Vol. 55, № 6, Novembro - Dezembro, 2005 
A recent study has shown that $24.7 \%$ of patients receiving vecuronium and $14.7 \%$ of those receiving rocuronium had TOF $<0.07$ in the PACU after attempt to revert neuromuscular block with pyridostigmine ${ }^{5}$.

Simpler stimulators allow for visual or tactile evaluation of muscle contraction only. However, several studies have suggested that even on experienced hands, it may be difficult to detect fatigue with those methods when $T_{4} / T_{1}>0,3^{10,11}$. Double burst stimulation helps visual or tactile detection of fatigue, but also have limitations ${ }^{11} \cdot T_{4} / T_{1}>0,3$ suggests that patient will not develop significant respiratory difficulty; however this is not enough to prevent gastric content aspiration or airways obstruction ${ }^{12}$. Swallowing difficulty disappears only with $\mathrm{T}_{4} / \mathrm{T}_{1}>0.9^{13}$. With TOF $<0.9$ on adductor pollicis, airways protection is impaired, increasing four to five times the risk of aspiration ${ }^{9}$. To assure normal muscular function and ventilatory regulation, TOF $>0.9$ on adductor pollicis should be reached before extubation ${ }^{9}$.

Many studies standardizing residual curarization as TOF < 0.7 have shown the incidence of $8 \%$ to $42 \%$ with vecuronium ${ }^{1}, 24 \%$ with mivacurium ${ }^{14}$, and up to $65 \%$ with atracurium. Considering that the safe TOF value for extubation is above 0.9 , the incidence of residual neuromuscular block is even higher than that found in those studies.

Subgroup $C$ has received the highest relative NMB dose (3.15DE95) as compared to subgroup R (2.2 DE95). This difference may be due to longer cisatracurium onset as compared to rocuronium, with higher relative dose to obtain satisfactory intubation conditions in a short period of time.

There has been a high incidence of postoperative neuromuscular block in both groups, without statistically significant differences in residual neuromuscular block between cisatracurium and rocuronium. The incidence of neuromuscular block in the PACU was $32 \%$ in subgroup C and $30 \%$ in subgroup R. The incidence of residual neuromuscular block was kept high, in levels equivalent to results obtained by recent studies.

There has been no statistically significant difference in NMB dose among RPOC patients, showing that bolus neuromuscular blocker has not determined residual neuromuscular block. Increasing cisatracurium dose prolongs deep neuromuscular block (without response to neurostimulation). However, at the beginning of spontaneous recovery, there is a similar profile, in spite of the difference in dose, thus allowing the calculation of the recovery period ${ }^{15}$. A similar study has concluded that atracurium dose is not associated to RPOC ${ }^{4}$. Another author has observed that higher vecuronium doses were associated to higher risk of RPOC ${ }^{1}$, however multiple NMB doses were used in this study.

There is statistically significant difference between NMB administration and TOF in both groups (Table IV). Shorter interval between NMB administration and procedure completion is related to higher incidence of residual neuromuscular block, so patients submitted to short procedures are at higher risk. Similar results were found with vecuronium ${ }^{1}$, atracurium ${ }^{4}$ and rocuronium ${ }^{5}$.
There was no statistical difference in neuromuscular block among patients receiving or not neostigmine (Table V). Neostigmine effectively antagonizes neuromuscular block ${ }^{16}$. There are controversies with regard to neostigmine doses needed for total reversal, because they depend on the drug, its dose, time elapsed since its administration and on the concomitant use of drugs which may interfere with neuromuscular blockers.

For all patients, time elapsed between neostigmine administration and residual neuromuscular block evaluation was above 10 minutes.

Mean neostigmine dose for cisatracurium and rocuronium subgroups was relatively low: $0.027 \mathrm{mg} \cdot \mathrm{kg}^{-1}$ and 0.029 $\mathrm{mg} . \mathrm{kg}^{-1}$, respectively. Mean neostigmine doses were not enough to revert neuromuscular block induced by cisatracurium and rocuronium, since there has been no statistically significant difference in TOF values among patients receiving or not the antagonist. Studies suggest that $0.05 \mathrm{mg} \cdot \mathrm{kg}^{-1}$ neostigmine is effective to revert vecuronium-induced neuromuscular block ${ }^{1,16}$

When patients were subdivided by neostigmine dose, it was observed that doses above $0.03 \mathrm{mg} . \mathrm{kg}^{-1}$ were effective to revert neuromuscular block and all patients receiving this dose arrived to the PACU with TOF above 0.9 (Table VI).

Many drugs may interact with neuromuscular blockers, changing blockade pattern. Our study was not sensitive to show this drug interaction since there has been no statistically significant difference in TOF values among patients using or not these drugs.

Neostigmine sub-doses and shorter procedures are associated to higher risk of RPOC. Careful and continuous observation in the PACU is critical even after reversal.

Objective neuromuscular function monitoring should always be adopted because it allows for the correct decision on the need for reversal, the reversal dose and the effectiveness of the antagonism.

Patients of both groups presented high incidence of residual neuromuscular block in the PACU. Postoperative residual curarization is still a problem, even with new intermediary action neuromuscular blockers.

\section{REFERÊNCIAS - REFERENCES}

01. Baillard C, Gehan G, Reboul-Marty J et al - Residual curarization in the recovery room after vecuronium. Br J Anesth, 2000;84: 394-395.

02. Berg H, Roed J, Viby-Mogensen J et al - Residual neuromuscular block is a risk factor for postoperative pulmonary complications. A prospective, randomised, and blinded study of postoperative pulmonary complications after atracurium, vecuronium and pancuronium. Acta Anaesthesiol Scand, 1997;41:1095-1103.

03. Oliveira AS, Bastos CO, Serafim MM et al - Avaliação do bloqueio neuromuscular residual na sala de recuperação pós-anestésica. Rev Bras Anestesiol, 1997;47:502-511.

04. McCaul C, Tobin E, Boylan JF et al - Atracurium is associated with postoperative residual curarization. $\mathrm{Br} \mathrm{J}$ Anaesth, 2002;89:766-769. 
05. Kim KS, Lew SH, Cho HY et al - Residual paralysis induced by either vecuronium or rocuronium after reversal with pyridostigmine. Anesth Analg, 2002;95:1656-1660.

06. Cammu G, Baerdemaeker L, den Blauwen N et al - Postoperative residual curarization with cisatracurium and rocuronium infusions. Eur J Anaesthesiol, 2002;19:129-134.

07. Tiret L, Desmonts JM, Hatton F - Complications associated with anesthesia - a prospective survey in France. Can Anaesth Soc J, 1986;33:336-344.

08. Griffth HR, Johnson GE - The use of curare in general anesthesia. Anesthesiology, 1942;3:418.

09. Eriksson LI - The effects of residual neuromuscular blockade and volatile anesthetics on the control of ventilation. Anesth Analg, 1999;89:243-251.

10. Gill S, Donati F, Bevan DR - Clinical evaluation of double burst stimulation: Its relatioship to train of four stimulation. Anesthesia, 1989;45:543.

11. Drenck NE, Ueda N, Olsen NV et al - Manual evaluation of residual curarization using double burst stimulation: a comparison with train of four. Anesthesiology, 1989;70:578-581.

12. Tardelli MA - Monitorização do Bloqueio Neuromuscular, em: Curso de Educação à Distância em Anestesiologia. São Paulo, Office Editora e Publicidade Ltda, 2002;177-190.

13. Eriksson LI, Sundman E, Olsson R - Funcional assessment of the pharynx at rest and during swallowing in partially paralyzed humans: simultaneous videomanometry and mechanomyography of awake human volunteers. Anesthesiology, 1997;87: 1035-1043.

14. Bevan DR, Kahwaji R, Ansermino JM et al - Residual block after mivacurium with or without edrophonium reversal in adults and children. Anesthesiology, 1996;84:362-367

15. Lepage JY, Malinovsky JM, Malinge $M$ et al - Pharmacodinamic dose-response and safety study of cisatracurium (51W89) in adult surgical patients during $\mathrm{N}_{2} \mathrm{O}-\mathrm{O}_{2}$ - opioid anesthesia. Anesth Analg, 1996;83:823-829.

16. Kopman AF, Zank LM, Neuman GG - Antagonism of cisatracurium and rocuronium block at a tactile Train-of-Four count of 2: should quantitative assessment of neuromuscular function be mandatory? Anesth Analg, 2004;98:102-106.

\section{RESUMEN}

Morais BS, Castro CHV, Teixeira VC, Pinto AS - Bloqueo Neuromuscular Residual después del Uso de Rocuronio o Cisatracúrio

JUSTIFICATIVA Y OBJETIVOS: El bloqueo neuromuscular residual en la sala de recuperación posanestésica (SRPA) es un fenómeno que puede aumentar la morbidez posoperatoria, con incidencia variando entre $0 \%$ y 93\%. La finalidad de este estudio fue evaluar la incidencia del bloqueo neuromuscular residual en la SRPA.

MÉTODO: Fueron estudiados 93 pacientes sometidos a cirugía general con el uso de cisatracúrio o rocuronio. Después de la admisión en la SRPA fue realizada la monitorización objetiva de la función neuromuscular (aceleromiografia - TOF-GUARD). El bloqueo neuromuscular residual fue definido como TOF $<0,9$.

RESULTADOS: Del total de 93 pacientes, 53 recibieron cisatracúrio y 40 rocuronio. Las características demográficas, duración del procedimiento y el uso de antagonista fueron comparables en ambos grupos. El bloqueo neuromuscular residual fue del $32 \%$ en el subgrupo C (cisatracúrio) y $30 \%$ en el subgrupo $R$ (rocuronio). El bloqueo neuromuscular residual no fue asociado a la dosis, a la edad, o al uso de antagonista, sin embargo presentó relación con la duración del procedimiento quirúrgico. En el subgrupo C el tiempo medio de cirugía fue de 135 minutos en los pacientes con bloqueo neuromusculary 161 minutos en los pacientes sin bloqueo, $p<0,029$. En el subgrupo $R$ el tiempo medio de cirugía fue 122 y 150 minutos, respectivamente, $p<0,039$.

CONCLUSIONES: Los pacientes de ambos grupos presentaron incidencia elevada de bloqueo neuromuscular residual en la SRPA. La curarización residual posoperatoria continúa un problema, lo mismo con los nuevos bloqueadores neuromusculares de acción intermediaria. Es de suma importancia la monitorización objetiva de todos los pacientes sometidos a la anestesia general con el uso del bloqueador neuromuscular. 